\title{
Player Perception of Delays and Jitter in Character Responsiveness
}

\author{
Aline Normoyle * \\ University of Pennsylvania
}

\author{
Gina Guerrero ${ }^{\dagger}$ \\ Clemson University
}

\author{
Sophie Jörg $\ddagger$ \\ Clemson University
}

\begin{abstract}
Response lag in digital games is known to negatively affect a player's game experience. Particularly with networked multiplayer games, where lag is typically unavoidable, the impact of delays needs to be well understood so that its effects can be mitigated. In this paper, we investigate two aspects of lag independently: latency (constant delay) and jitter (varying delay). We evaluate how latency and jitter each affect a player's enjoyment, frustration, performance, and experience as well as the extent to which players can adjust to such delays after a few minutes of gameplay. We focus on a platform game where the player controls a virtual character through a world. We find that delays up to $300 \mathrm{~ms}$ do not impact the players' experience as long as they are constant. When jitter was added to a delay of $200 \mathrm{~ms}$, however, the lag was noticed by participants more often, hindered players' ability to improve with practice, increased how often they failed to reach the goal of the game, and reduced the perceived motion quality of the character.
\end{abstract}

CR Categories: I.3.7 [Computer Graphics]: Three-Dimensional Graphics and Realism-Animation K.8.0 [Personal Computing]: General-Games;

Keywords: virtual characters, digital games, responsiveness, controller latency, control lag, jitter

\section{Introduction}

The responsiveness of a character, known as response lag or input lag, is the time between the user's input and its corresponding response. Input lag can be due to several reasons: network de-

*e-mail:alinen@seas.upenn.edu

†e-mail:gguerre@clemson.edu

‡e-mail:sjoerg@clemson.edu lays, lags introduced by software and hardware (e.g., display lag), server processing speed, or complex computations of AI or character movements [Jehaes et al. 2003; McCann and Pollard 2007].

Response lag is known to negatively affect players' game experience. The efforts of organizers of online multiplayer competitions or of gamers to reduce delays demonstrate the importance attributed to this topic. Gamers usually opt for local game servers to reduce lag [Jehaes et al. 2003]. UGC (United Games Clans), which offers competitive gameplay for the team-based multiplayer online games Team Fortress and Dota, has a set of rules concerning servers and server issues. For example, if the average team ping between battling teams differs by more than $100 \mathrm{~ms}$, the disadvantaged team can request to change server [UGC 2014].

Understanding when and how latency affects the game experience is crucial for ensuring a consistently good game experience for the player because such knowledge enables us to develop better algorithms for hiding such latency from the player. In this paper, we study the amount of lag tolerable in cases where delays are either constant or varying over time. The variation in the delay is referred to as jitter.

Using a platform game (see Figure 1), we investigate how latency and jitter independently affect the player's experience. We focus on casual players and a scenario where the participants are not aware of the experiment's goal and can not compare different types of latencies or jitter. We analyze the effects of latency and jitter on five aspects of gameplay: ease of control, player enjoyment, player frustration, player performance, and perceived character and game quality. We also look for thresholds of latency and jitter which are tolerable for players. Lastly, we investigate the ability of the player to notice the delay and to adapt to latency and jitter with practice.

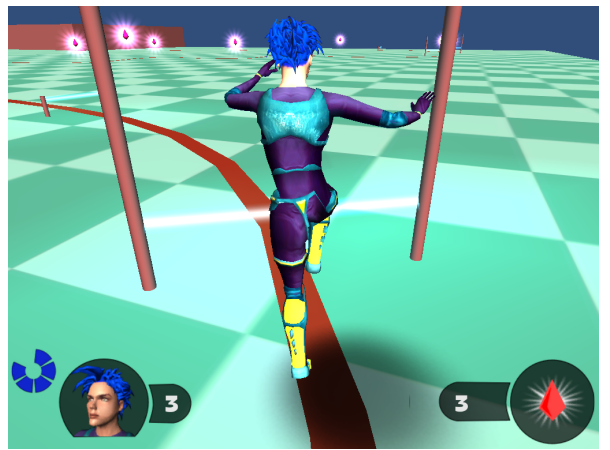

Figure 1: Screenshot from level 1 of the platform game. The player has to follow a path (red line), avoid lasers, and collect gems.

Specifically, we perform two sets of controlled experiments which evaluate latency and jitter independently using the experimental setup described in Jörg et al. [2012]. In the first experiment, we simulate varying levels of latency with no jitter: $0 \mathrm{~ms}, 50 \mathrm{~ms}, 100 \mathrm{~ms}$, $200 \mathrm{~ms}, 300 \mathrm{~ms}$, and $500 \mathrm{~ms}$. In the second experiment we simulate varying levels of jitter with a fixed latency: $50 \mathrm{~ms}, 100 \mathrm{~ms}$, and $150 \mathrm{~ms}$, all with a $200 \mathrm{~ms}$ base delay.

We find that latencies as large as $300 \mathrm{~ms}$ barely affect the player experience, and only at $500 \mathrm{~ms}$ does the player experience alter significantly. However, when jitter with a variance of $150 \mathrm{~ms}$ is added 
to an average latency of $200 \mathrm{~ms}$, the experience is also significantly altered with more players noticing the delay than for latencies up to $500 \mathrm{~ms}$. Jitter and latency can both degrade the player's performance. While both degrade the player's ability to jump across platforms, only latency reduces the player's accuracy when following a path. Finally, the higher the constant latency, the more players feel their ability to control the character improves with practice whereas this effect does not occur in the presence of jitter. Additionally, latency and jitter reduces the perceived motion quality of the character, with participants citing poor controls as a primary reason for their low ratings.

\section{Related Work}

Previous studies investigated the effect of latency on gameplay. The threshold latency over which a game becomes unplayable varies. Claypool and Claypool [2006] identified different categories of games and analyzed the amount of tolerable latency for each. Their findings suggested that a game requiring fast paced, high precision actions, such as with first person shooters and racing games, should have a maximum latency of $100 \mathrm{~ms}$. Third-person games, however, can tolerate delays of up to $500 \mathrm{~ms}$. A study using Quake 3 [Armitage 2003] showed that players actively prefer Quake 3 servers with less than 150 to $180 \mathrm{~ms}$ delay. Beigbeder et al. [2004] found that latencies as low as $100 \mathrm{~ms}$ can significantly degrade performance in Unreal Tournament 2003 and latencies over 150ms make the game feel sluggish. In contrast, Dick et al. [2005], when comparing player performance in Unreal Tournament, Counter-Strike, and Need for Speed, demonstrated that although a $150 \mathrm{~ms}$ delay was noticeable, a 500ms delay was still playable and did not decrease performance.

Studies most often analyze performance and playability. In previous work, we investigate different aspects of the game experience such as the player's perceived ease of control, their enjoyment, their frustration, and their ratings of motion quality when playing a 3D platform game [2012]. We observed that an average of $150 \mathrm{~ms}$ of delay significantly reduced the player's perceived ease of control, their performance, as well as their satisfaction with it.

In the previously cited studies, latency and jitter are usually not considered individually and the reported latencies include some jitter. On public servers jitter and latency are highly correlated [Armitage and Stewart 2004]. Other studies do investigate latency and jitter separately or even focus on jitter. Beznosyk et al. [2011] observed that in the cooperative, multiplayer game Little Big Planet 2 delays over $100 \mathrm{~ms}$ significantly decrease performance. They also noted that although players' performance decreased with jitter, players did not perceive this as disturbing.

At low levels, jitter might not be perceived by players. In Unreal Tournament 2003, Quax et al. [2004] found that jitter values up to $95 \mathrm{~ms}$ did not impact players whereas latencies starting at $60 \mathrm{~ms}$ did. Pavlovych and Gutwin's study [2012] examined the effect of latency and jitter in a mouse-based accuracy task and observed that user accuracy was greatly affected by a $60 \mathrm{~ms}$ jitter with $170 \mathrm{~ms}$ of average latency. Another study investigating delay, jitter, and packet loss found that jitter only had a minor influence on the win probability as well as the score rates of two AI bots [Bredel and Fidler 2010]. They took into account jitter up to $50 \mathrm{~ms}$ with a delay of 100ms. Amin et al. [2013] observed a drop in their averaged ratings between jitters of $100 \mathrm{~ms}$ and $250 \mathrm{~ms}$ for subjects playing Call of Duty Modern Warfare 2.

In this work, we evaluate the extent to which latency and jitter change a player's game experience. In contrast to previous research, our participants did not know that our study investigated the effects of latency and jitter and were not able to compare different conditions. To separate effects due to latency and jitter, we first analyze the effect of increasing constant delays and then analyze the effect of adding jitter to a delay of $200 \mathrm{~ms}$. Lastly, in addition to analyzing players' performance and the noticeability of the delay, we analyze how delay affects players' frustration, enjoyment, and perception of the character.

\section{Method}

We investigate the impact of latency and jitter on the player's experience, closely following Jörg et al.'s [2012] experimental method.

\subsection{Hypotheses}

Based on previous literature, we expect both latency and jitter to negatively impact the player's experience. Although we expect larger amounts of latency or jitter to have more impact than smaller amounts, the thresholds at which latency and jitter effect different aspects of the player's experience is unclear.

Furthermore, we hypothesize that a player is able to adapt to constant amounts of delay with practice (referred to as a learning ef$f e c t)$ but that jitter will hinder this effect.

1. H1 - Control:

(a) The player finds it more difficult to control the character as latency increases.

(b) The player finds it more difficult to control the character as jitter increases.

(c) The player can adapt to constant latencies with practice (learning effect). Thus, as latency increases, we expect the improvement of players' ratings of the ease of control between level 1 and level 3 to increase.

(d) The player's ability to improve with practice does not increase as jitter increases (reduced learning effect).

2. H2 - Enjoyment:

(a) The player's enjoyment decreases as latency increases.

(b) The player's enjoyment decreases as jitter increases.

3. H3 - Frustration:

(a) The player's frustration increases as latency increases.

(b) The player's frustration increases as jitter increases.

4. H4 - Performance:

(a) The player's performance decreases as latency increases.

(b) The player's performance decreases as jitter increases.

(c) The player can adapt to constant latencies with practice (learning effect). Thus, as latency increases, we expect the improvement in players' performance between level 1 and level 3 to increase.

(d) The player's ability to improve her performance with practice does not increase as jitter increases (reduced learning effect).

5. H5 - Perception of character and game:

(a) The player's perception of the character and the game will not change as latency increases. 
(b) The player's perception of the character and the game will not change as jitter increases.

To test these hypotheses we carry out two experiments in which we ask players to complete three levels of a 3D platform game. The player steers a human character through a futuristic environment collecting gems, avoiding lasers, and jumping between platforms.

\subsection{Stimuli}

To explore the effects of both latency and jitter individually on the player's experience, we design two experiments: a latency experiment and jitter experiment. For both experiments, we use the game developed by Jörg et al. [2012] as a baseline and only change the character controller. We briefly describe the main features of that game. Scenes from the gameplay can be seen in the accompanying video.

The game is created in Unity and consists of 3 levels. Level 1 and level 3 are exactly the same, which allows us to investigate the possible learning effect. In level 1 and level 3, we ask players to follow a path as quickly and as accurately as possible. In level 2 , which is designed to be more difficult, players are asked to traverse an environment containing elevated platforms from which the player can fall and die if jumps are not timed correctly. In every level, players must avoid lasers which will reduce the player's health while collecting gems.

The player traverses the environment using a gamepad to steer a female, humanlike character who can stand, jump, and jog. The character transitions immediately between motions without blending to ensure that transitions are immediately visible. A third-person camera automatically follows the character (chosen to accommodate players with less game experience who would find simultaneously steering the character and camera difficult). The game starts in a tutorial area where the player can become accustomed to the controls before starting the experiment.

In our latency experiment, we test six conditions simulating a constant latency: $0 \mathrm{~ms}, 50 \mathrm{~ms}, 100 \mathrm{~ms}, 200 \mathrm{~ms}, 300 \mathrm{~ms}$, and $500 \mathrm{~ms}$. Those values were chosen to cover a typical range of latency values from 50-300ms [Armitage and Stewart 2004] as well as one extreme condition of $500 \mathrm{~ms}$. To simulate a constant delay, we timestamp and buffer the player's gamepad input into a FIFO queue. Each frame, we process all commands whose elapsed wait time is greater than the given delay. As a result, all input commands are processed in the correct order, only with a fixed delay between the input time and the processing time.

In the jitter experiment, we test four conditions with a fixed average latency but with increasing variance in the delay. We specify a mean delay of $200 \mathrm{~ms}$ combined with either a $50 \mathrm{~ms}, 100 \mathrm{~ms}$, or $150 \mathrm{~ms}$ jitter. A mean of $200 \mathrm{~ms}$ was chosen because it was the smallest delay deemed large enough to test a range of varying jitter. To simulate latency with jitter, we sample delay latencies from a given distribution. For each delay interval, we suspend execution of the player's input until the delay time completes. During suspension, the player's input is again buffered in a FIFO queue and after suspension completes, processed in order. We use a $\operatorname{Gamma}(k, \theta)$ distribution to model delay jitter, where $k$ is a shape parameter and $\theta$ is a scale parameter (mean $=k \theta$ and variance $=k \theta^{2}$ ). A Gamma distribution allows us to model the distributions with right-tailed asymmetries typical of network latencies [Armitage 2002]. From the player's perspective, the effect of the jitter is a character having sporadic short periods of unresponsiveness. Resulting distributions are shown in Figure 2. A summary of our conditions for both experiments in represented in Table 1.
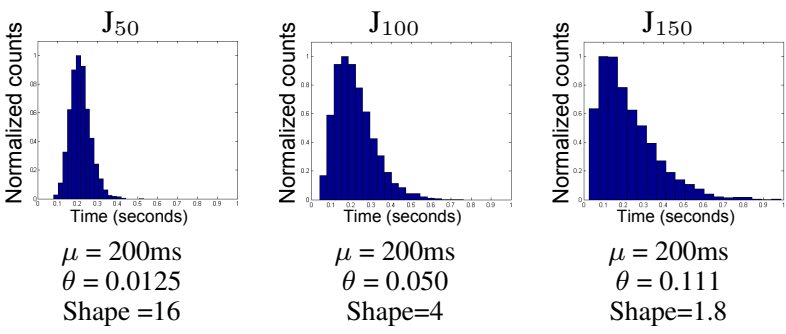

Figure 2: Latency distributions for the jitter experiment. We model latencies using a gamma distribution with the shape parameter $k$ and the scale parameter $\theta$. The shape parameter corresponds to the amount of asymmetry in the delay. Each distribution has the same mean $\mu=k \theta$, but we vary the spread using the scale parameter $\theta$, which results in a distribution with a longer right tail.

Latency Experiment

\begin{tabular}{lll}
$\begin{array}{l}\text { condition } \\
\text { name }\end{array}$ & $\begin{array}{l}\text { latency } \\
\text { in ms }\end{array}$ & $\begin{array}{l}\text { jitter } \\
\text { in } \mathrm{ms}\end{array}$ \\
\hline $\mathrm{L}_{0}$ & 0 & 0 \\
$\mathrm{~L}_{50}$ & 50 & 0 \\
$\mathrm{~L}_{100}$ & 100 & 0 \\
$\mathrm{~L}_{200}$ & 200 & 0 \\
$\mathrm{~L}_{300}$ & 300 & 0 \\
$\mathrm{~L}_{500}$ & 500 & 0
\end{tabular}

Table 1: Latency and jitter values of all conditions for the two experiments. Notice that $L_{200}$ and $J_{0}$ are the same condition. The answers from the same participants were used for $L_{200}$ and $J_{0}$ in the evaluations. We use the term $L_{200}$ or $J_{0}$ depending on which experiment we refer to.

\subsection{Participants}

A total of 89 participants took part in the study: 10 in each condition, except for $\mathrm{L}_{300}$ which had 9 participants. The participants, students and researchers from a diversity of disciplines, were recruited through flyers and class announcements. There were between 3 and 5 female participants in each condition. They were rewarded $\$ 5$ for their participation.

\subsection{Procedure}

We followed Jörg et al.'s [2012] procedure as closely as possible, adapting it to our IRB protocols and adding questions to gain further insights. We used a between-group design so that users could not compare different conditions. Thus, each participant played through all three levels in the same condition and experienced only one type of delay.

Participants were given an informational letter regarding the study, and were asked to answer basic demographic questions as well as their experience with digital games and virtual characters on a 7 point scale. They were then given instructions describing the controls of the game and were asked to play through level 1 , level 2, and level 3 (which is the exact same as level 1). Once the level loaded, they were given verbal instructions as to the goal of the level. For level 1 and 3, they were to follow a path as quickly and as accurately as possible. For level 2, the instructions were to reach the big gem at the end of the level.

During game play, we recorded game metrics to evaluate the player's performance. After each level, participants were asked to answer four questions on 7-point Likert scales specific to the level last played: "How much did you enjoy playing this level?", "How did you feel about your performance in this level?", "How diffi- 
cult/easy was it to control the character in this level?", and "How would you rate the quality of the motions in this level?".

At the end of the full experiment, they were furthermore asked to rate how much specific attributes applied to the game (e.g., entertaining, challenging, repetitive) and to the character (e.g., agile, humanlike, sympathetic). They were asked why they rated the motions as high or low quality, what they liked or disliked in the game, and what they thought the purpose of the study was. They were also given time to write down any comments they may have. Afterwards, the purpose of the study was explained to them, and we asked participants who did not explicitly mention a delay if they felt that the character was responsive by asking them if they felt that the character acted as soon as they pressed a button. Finally, they were asked to sign a consent form so that we may use their data. All in all, the study was about 30 minutes long.

\section{Results}

The results to the four questions asked after each level were analyzed using a repeated measures ANOVA with the factors Level (within-subjects variable with three values) and Condition. The factor Condition is called Latency (between-subjects variable with six different values $\mathrm{L}_{0}-\mathrm{L}_{500}$ ) in the latency experiment, where we vary the delay between the control signal and the reaction of the virtual character, and Jitter (between-subjects variable with four different values $\mathbf{J}_{0}-\mathbf{J}_{150}$ ) in the jitter experiment, where we change the variance in the delay. When a significant effect was found, we used Newman-Keuls post hoc tests to determine the reasons for the effect.

\subsection{Control}

We found a main effect of Latency with $F(5,53)=5.9$ and $p<0.001$ for how difficult it was to control the character in each level. The post hoc test shows that the effect is caused by the $\mathrm{L}_{500}$ condition, but a trend to lower ratings starts after $\mathrm{L}_{200}$ (see Figure 4). With $\mathrm{p} \approx 0.1$ there is no significant effect of Jitter $(\mathrm{F}(3,36)=2.18)$. However, a downward trend can be observed in Figure 4. Based on our data, we can support hypothesis $\mathrm{H} 1-\mathrm{a}$, that the player finds it more difficult to control the character as latency increases but we can not conclusively support H1-b, that the player finds it more difficult to control the character as jitter increases.

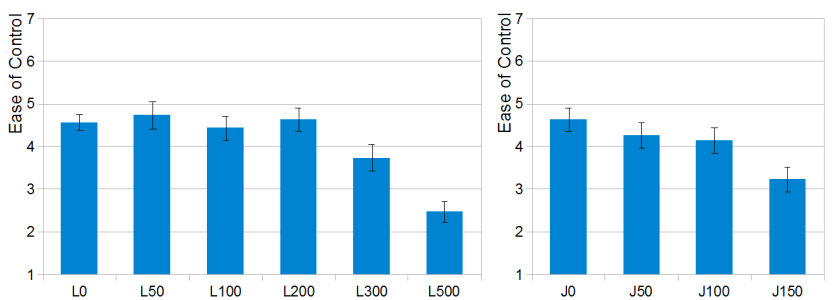

Figure 4: Ease of control rated on a 7-point Likert scale with 1 "very difficult" and 7 "very easy". There is a significant effect of Latency on the perceived ease of control due to the $L_{500}$ condition (left) and a trend that steering the character is perceived more difficult for increasing jitter values (right). Error bars represent one standard error of the mean in all graphs.

Main effects of Level are found in both experiments with $\mathrm{F}(2,106)=28.9$ and $\mathrm{p}<0.001$ for the latency experiment and $\mathrm{F}(2,72)=28.9$ and $\mathrm{p}<0.001$ in the jitter experiment. In both experiments, participants find it most difficult to control the character in level 2 and easiest in level 3 with all differences being significant (Figure 6).
To evaluate if the player thinks he/she can adapt to increasing constant latencies but not in the presence of increasing jitter, we compare across conditions how the ease of control was rated after level 1 was played for the first time and after it was played for the second time (level 3). To this aim, we generate a new variable as the difference between the control ratings in level 3 and in level 1 for each participant. A one-way ANOVA for each experiment with the respective Condition as the dependent variable results in a significant effect of Latency $(\mathrm{F}(5,53=2.87), \mathrm{p}<0.05)$ but no effect for Jitter $(\mathrm{F}(3,36=1.00), \mathrm{p} \approx 0.4)$. A Newman-Keuls post hoc test shows that the significant effect is due to the value in the $\mathrm{L}_{500}$ condition being significantly higher than the values in the $\mathrm{L}_{50}$ and $\mathrm{L}_{100}$ conditions. Those results support hypothesis H1-c and H1-d, that the player's improvement increases with increasing constant latencies but not with increasing jitter (Figure 5). Note that participants did not know the purpose for the experiment nor that level 1 and 3 were the same. Thus, players may have believed that the controller was different between level 1 and level 3 .

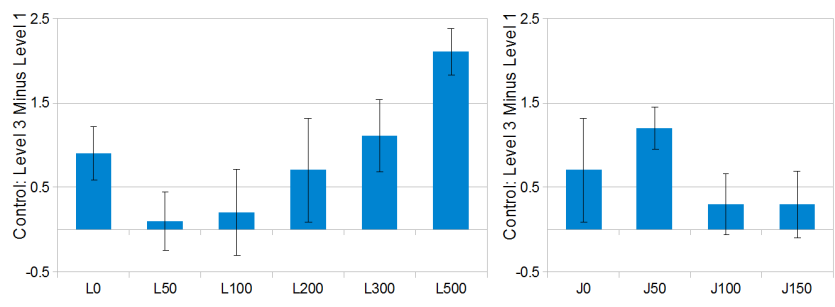

Figure 5: Differences between the ratings of the ease of control for level 3 and level 1. These results support a perceived learning effect for increasing latency values but not for increasing amounts of jitter.
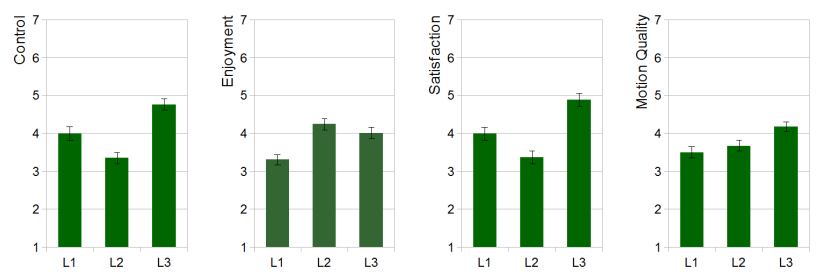

Figure 6: Ratings for each level for control, enjoyment, satisfaction, and motion quality averaged over all conditions.

\subsection{Enjoyment}

How much participants enjoyed playing each level did not yield a significant effect of Condition, neither for the latency nor for the jitter experiment (Figure $7, \mathrm{p} \approx 0.6$ and $\mathrm{p} \approx 0.8$ for latency and jitter, respectively). We can therefore not support $\mathrm{H} 2$-a nor $\mathrm{H} 2-\mathrm{b}$. In both experiments, there were significant main effects of Level $(\mathrm{F}(2,106=22.3)$ and $\mathrm{F}(2,72=12.8), \mathrm{p}<0.001)$ as level 1 was enjoyed significantly less than level 2 and level 3 each time and (Figure 6).

\subsection{Frustration}

To estimate frustration, we evaluate participant's ratings of their own performance in each level on a 7-point Likert scale ( 1 - very unsatisfied to 7 - very satisfied). We do not find any significant effects for Latency or Jitter; however, the results for Jitter $(\mathrm{F}(3,36)=2.22, \mathrm{p} \approx 0.1)$ indicate a downward trend (Figure 8). We can therefore not support $\mathrm{H} 2-\mathrm{a}$ nor $\mathrm{H} 2-\mathrm{b}$, that the player's enjoyment decreases as latency or jitter increase. In both experiments, there is a main effect of Level (latency experiment: $F(2,106)=26.5$, 


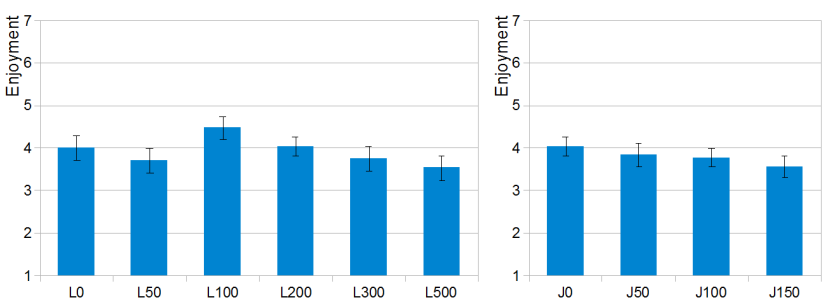

Figure 7: Ratings of how much participants enjoyed playing the levels on a 7-point Likert scale with 1 "not at all" and 7 "a lot". There are no significant differences.

$\mathrm{p}<0.001$; jitter experiment: $\mathrm{F}(2,72)=25.8, \mathrm{p}<0.001)$. Participants are most satisfied with their performance after level 3 and least after level 2 with all differences being significant (Figure 6).

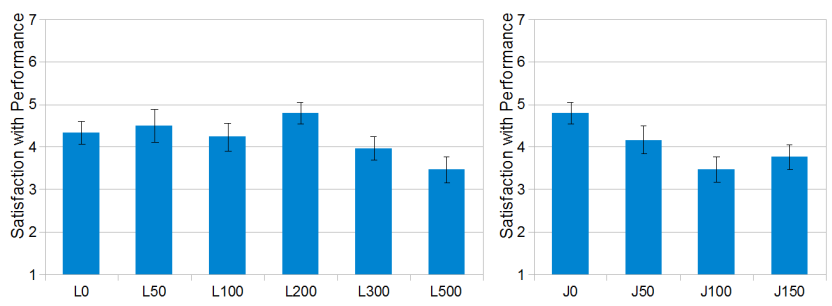

Figure 8: Ratings of how satisfied players were with their performance on a 7-point Likert scale with 1 "very unsatisfied" and 7 "very satisfied". There are no significant differences.

\subsection{Performance}

To evaluate performance, we measured several metrics during game play: the number of gems collected, the amount of lost health, the number of lives lost, the position of the character every half second, the amount of time spent in the tutorial area in level 1 and 3, and the amount of time needed to finish the level.

We find a conspicuous issue for the second level when analyzing our metrics: a considerable number of the participants (36 out of 89) lose all of their 7 lives without reaching the goal in level 2, mostly by falling off the platforms. This number varies highly between conditions (see Figure 9). For example, in condition $\mathrm{L}_{0}$ all 10 players reach the goal whereas in condition $L_{500}$ and condition $J_{150}$ only 2 and 3 players, respectively, out of 10 complete the level. A Kruskal-Wallis test shows that those differences are significant for the latency experiment $(\mathrm{p}<0.05)$ with $\mathrm{L}_{0}$ and $\mathrm{J}_{0}$ being significantly different from $\mathrm{L}_{500}$ and $\mathrm{J}_{150}$ and the jitter experiment $(\mathrm{p}<0.05)$, respectively. Unsurprisingly, we find nearly identical results when analyzing the amount of lives lost in level 2 for each condition.

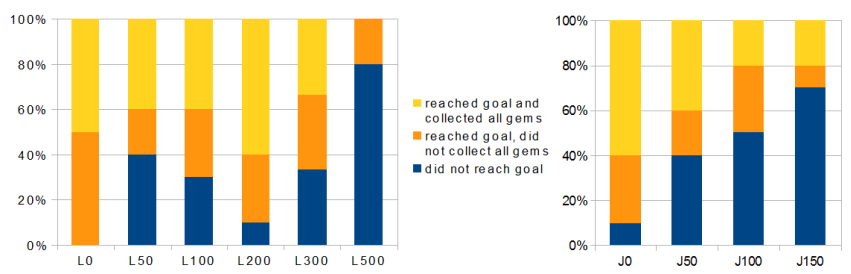

Figure 9: Percentages of players who did not reach the goal in level 2 (dark blue), who did reach the goal but did not collect all of the gems (orange), and who did reach the goal and collected all of the gems (yellow).

If some of the participants in a specific condition did not reach the end of level 2, this means that they had less chances to collect gems or lose health from laser beams. We therefore exclude level 2 from parts of the performance analysis. We find no significant effects of Latency when looking at the loss of health in level 1 and level 3 (repeated measures ANOVA). The results failed to reach significancy in the jitter experiment with $\mathrm{p}<0.1$ with the average amount of health lost increasing with increasing jitter except for $J_{150}$. In both experiments we found a main effect of Level as players lost significantly less health in level 3 (latency experiment: $F(1,53)=6.6$, $\mathrm{p}<0.05$; jitter experiment: $\mathrm{F}(1,36)=8.9, \mathrm{p}<0.01)$.

The accuracy when following a path in level 1 and level 3 showed a main effect of Latency $(F(5,53)=9.2, p<0.001)$ as players followed the path less accurately in $\mathrm{L}_{500}$. The accuracy of path following was not affected by increased jitter (see Figure 10). Similarly, for the time to reach the goal, there was a main effect of Latency $(\mathrm{F}(5,53)=2.52, \mathrm{p}<0.05)$ due to participants in $\mathrm{L}_{500}$ and no effect of Jitter. One way ANOVAs show no significant effects of the time spent in the tutorial area on Latency or on Jitter.
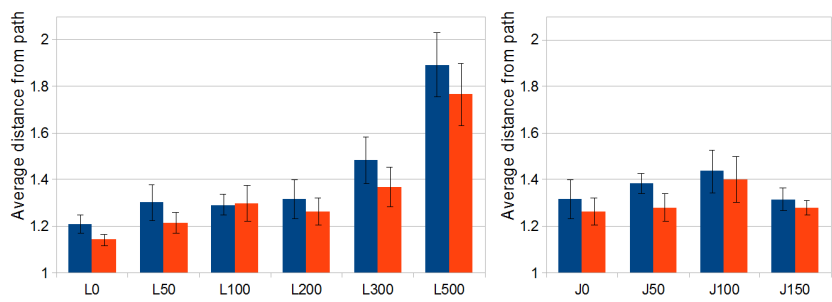

Figure 10: Average distance from the path in level 1 (dark blue) and level 3 (red). One unit of length in our game environment is approximately one foot. We found a main effect of Latency but no effect for Jitter.

Regarding path accuracy, we also found a main effect of Level with players following the path more accurately in level 3 than in level 1 (latency experiment: $\mathrm{F}(1,53)=11.0, \mathrm{p}<0.01$; jitter experiment: $\mathrm{F}(1,36)=9.9, \mathrm{p}<0.005)$. However, analyzing a new variable, the accuracy in level 3 minus the accuracy in level 1, does not lead to any significant results. The same holds true for the time to reach the goal. The accuracy when following a path is illustrated using maps in Table 3.

Based on a metric such as the number of lives lost, we can definitely support H4-a and H4-b. However, because a decrease in performance can not be observed in all metrics, particularly regarding path accuracy and the time to reach the goal, we cannot support hypothesis H4-c and also H4-d, that players' improvement in performance increases with practice with increasing constant latency but not when there is jitter.

\subsection{Perception of the Character and the Game}

As in Jörg et al. [2012], we do not find significant differences for the attribute ratings describing the game or the character. However, the ratings for motion quality significantly decrease with increasing latency and jitter (Figure 11, latency experiment: $\mathrm{F}(5,53)=2.5$, $\mathrm{p}<0.05$; jitter experiment: $\mathrm{F}(3,36)=25.8, \mathrm{p}<0.05)$. The post hoc test shows that the motion quality was rated significantly lower in $\mathrm{L}_{500}$ than in $\mathrm{L}_{0}$ and that $\mathrm{J}_{150}$ was rated significantly lower than $\mathbf{J}_{50}$. A main effect of Level is also determined for both experiments (latency experiment: $\mathrm{F}(2,106)=15.6, \mathrm{p}<0.001$; jitter experiment: $\mathrm{F}(2,72)=11.3, \mathrm{p}<0.001)$. In the both experiments level 3 is rated as having a significantly higher motion quality than the other levels. Additionally, in the latency experiment level 2 is rated to have a significantly higher motion quality than level 1 (Figure 6). 


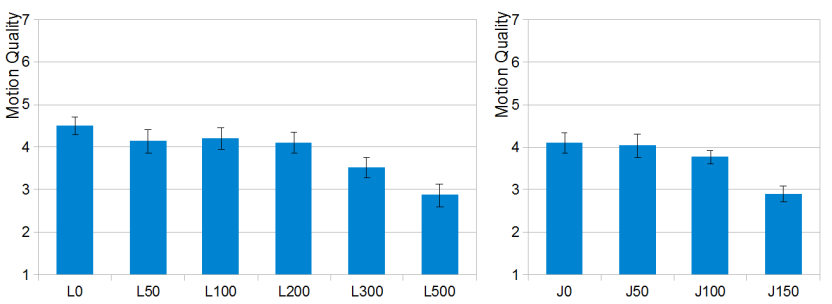

Figure 11: Ratings for the quality of the motions on a 7-point Likert scale with 1 "very low quality" and 7 "very high quality". There are main effects of Latency and Jitter with $L_{500}<L_{0}$ and $J_{150}<J_{50}$.

Our results do not support H5-a and H5-b, as the perception of the character and the game change as latency increases and as jitter increases. Note that the motion quality was identical across levels and across conditions. However, participant comments revealed that controls were often factored into ratings of quality. in particular, 56/89 participants specifically mentioned the controls when justifying their rating for poor motion quality, more so than factors related to realism and movement (31), the jump motion (27), or the motion transitions (4).

\subsection{Noticing a Delay}

Based on the participant's comments, we determined whether players noticed a delay (Figure 12). A Kruskal-Wallis test showed that the differences failed to reach significancy in the latency experiment with $\mathrm{p} \approx 0.1$. The constant $300 \mathrm{~ms}$ delay in $\mathrm{L}_{300}$ was only noticed by 4 out of 9 participants and even the large constant $500 \mathrm{~ms}$ delay in $\mathrm{L}_{500}$ was not perceived by every participant ( 8 out of 10 ). The effect was significant, however, for the jitter experiment (KruskalWallis test with $\mathrm{p}<0.05$ ). While the $50 \mathrm{~ms}$ and $100 \mathrm{~ms}$ variance was only noticed by some of the players, every single participant in $\mathbf{J}_{150}$ perceived the $200 \mathrm{~ms}$ delay with $150 \mathrm{~ms}$ variance.
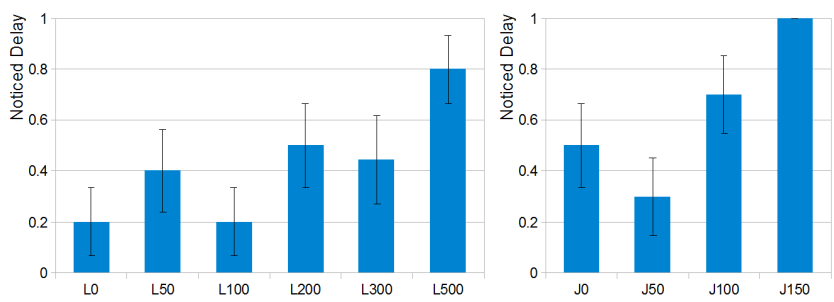

Figure 12: Ratios of participants in each condition who reported to notice a delay in the controls, that the character was not responsive, or that the character did not act as soon as they pressed a button.

\section{Discussion}

Unlike Jörg et al. [2012], which evaluated a single 150ms delay against no latency, this work focuses on the effects of constant versus varying delays. As expected, many of our findings are consistent with the previous experiment: the effect of Level on improved path following, enjoyment, and frustration did not change; and delay had no effect on enjoyment.

However, this paper sheds new light on several previous findings. Regarding ease of control, the previous experiment showed a significant effect of delay, which we do not see in this study until after $300 \mathrm{~ms}$ of latency, or as a downward trend in the jitter experiment where $p \approx 0.1$. Similarly, the previous experiment showed a significant increase in frustration in the delay condition whereas in

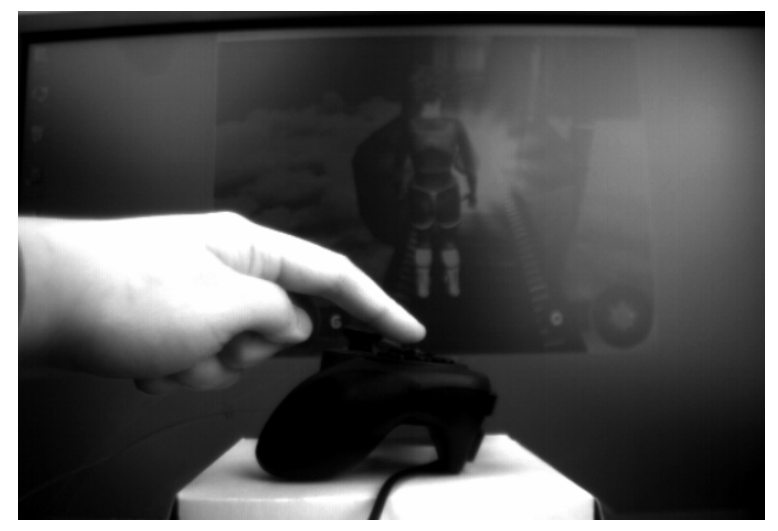

Figure 13: A high speed camera was used for accurate latency measurements. We determined the time between the push of the gamepad button and the character's response.

this experiment, we found no effect of latency but a trend for jitter. We believe these differences result from not carefully controlling for jitter in the previous experiment. In other words, our previous $150 \mathrm{~ms}$ delay contained both latency and jitter.

Regarding performance, in our current experiment, jitter increased the number of lives lost in level 2, but did not affect the accuracy when following the path in levels 1 and 3 , whereas latency affected both. We believe that the latency in the previous experiment was too low to have an effect on path accuracy. However, our current experiment shows that higher latencies affect path following while jitter does not. Conversely, jitter interferes with the player's ability to time jumps or avoid lasers resulting in more deaths and health loss. Thus, we believe that the significant increase in health lost and lives lost in the previous experiment was due to jitter. Also, our new experiments provide thresholds for when latency is noticeable (none of the participants of our previous experiment explicitly mentioned a delay): either for very large latencies or in the presence of jitter. Regarding motion quality, we only saw a trend earlier, whereas we now find the differences in ratings to be significant.

\subsection{Latency Accuracy}

We log latency from the time between when we receive the user's input and when the character begins to respond. However, there can still be small fluctuations in the delay due to frame update rates. We tracked those fluctuations by performing random sample tests, and found them to be negligible. As an example, for a randomly selected individual participant's gameplay through the first level, out of the over 85,000 inputs more than $80 \%$ were processed within $2 \mathrm{~ms}$ and over $96 \%$ within $3 \mathrm{~ms}$ of the input time.

Because our logs only capture the delay from when our application received an input event, our definition of latency excludes the total latency starting from when the user presses the gamepad. To estimate this baseline latency, we use a high speed camera to record the time between a button press and the character's response. We recorded the controller and monitor in the same field of view at 1000 frames per second (Figure 13). Using the $L_{0}$ condition, which had no delay and no jitter, we recorded the virtual character transitioning to jumping from either walking or idling. In this condition, the recorded latency is zero. When computing the baseline latency, we consider the entire interval between when the jump button is fully pressed to when the player lifts her finger off the button.

We performed six tests under different conditions (such as while colliding into a gem versus not) in both the level 1 and level 2 en- 
vironments. From button press, the average latency was $26.7 \mathrm{~ms}$. From finger lift, the average latency was $51.2 \mathrm{~ms}$. At $60 \mathrm{fps}$, this corresponds to an additional delay of 1-3 frames on top of the latencies recorded in our logs. Again, we believe this overhead to be negligible. First, because additional latency is usually not accounted for in related work, our results can still be compared to other studies. Secondly, this additional latency is present in all our experiment conditions, making each comparable to each other. Lastly, because we do not see significant effects for latencies below $300 \mathrm{~ms}$, players appear oblivious to this overhead.

\section{Conclusion and Future Work}

In this paper, we systematically test the effects of latency versus jitter on players' enjoyment, their ability to control the character, their performance, their frustration, and their opinions of the character and game. Our results show that responsiveness indeed contributes to players' game experience, but that it's the nature of the latency, not just the amount of latency, that is important.

Similarly to previous work, we attempted to find a threshold over which a constant latency becomes unplayable. We expected to see a steady downward trend as latency increases, but instead saw that a constant latency had very little affect, until it was set very high (500ms). In fact, players seem able to adapt to a constant delay and, in general, often did not notice that a delay was even present. For example, the constant delay of $300 \mathrm{~ms}$ was detected by less than half of the players of that condition. However, this was not the case with jitter. When jitter was added to a delay of $200 \mathrm{~ms}$, it was noticed by all participants. These findings suggest that to hide latency from players in networked games, the most effective strategy is to hide the jitter, even if that means introducing a larger constant latency. In other words, it is better to have a larger constant latency than one which is shorter on average but has more variability.

However, to fully confirm this statement, we need to understand in detail what parts of jitter are responsible for the observed effects: Is it the fact that there is some variation at all or is it due to individual inputs with high delays that exceed a threshold (such as 500ms)? Further work will be required to completely understand how jitter affects the player experience. Also, we would like to verify our findings in different types of games, particularly those which require strict timing, as in a racing game or competitive multiplayer shooter. We are furthermore interested in other factors which may affect the noticeability of delays, such as sound or visual effects (e.g. capes and particle trails). Lastly, we would like to study the effect of animation quality, particularly how players respond to a loss of responsiveness when it enables more realistic and higher quality motions.

\section{Acknowledgements}

We wish to thank Linh Thi Xuan Phan for her advice regarding network latency characteristics, Adam Hoover and Ryan Mattfeld for lending their high-speed camera, our participants for their time, everyone who was involved in recruiting participants for their help, and our reviewers for their valuable comments.

\section{References}

Amin, R., Jackson, F., Gilbert, J. E., Martin, J., And SHAW, T. 2013. Assessing the impact of latency and jitter on the perceived quality of call of duty modern warfare 2. In HumanComputer Interaction: Users and Contexts of Use, HCI'13, 97106.
Armitage, G., And Stewart, L. 2004. Limitations of using real-world, public servers to estimate jitter tolerance of first person shooter games. In SIGCHI Advances in Computer Entertainment Technology, ACE '04, 257-262.

Armitage, G., 2002. Lag over 150 milleseconds is unacceptable. http://gja.space4me.com/things/ quake3-latency-051701.html. Retrieved May 2014.

Armitage, G. 2003. An experimental estimation of latency sensitivity in multiplayer Quake 3. In IEEE International Conference on Networks. ICON 2003, 137-141.

Beigbeder, T., Coughlan, R., Lusher, C., Plunkett, J., Agu, E., ANd Claypool, M. 2004. The effects of loss

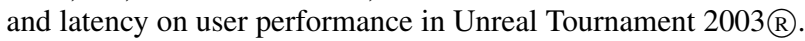
In SIGCOMM Workshop on Network and System Support for Games, NetGames '04, 144-151.

Beznosyk, A., Quax, P., Coninx, K., and Lamotte, W. 2011. Influence of network delay and jitter on cooperation in multiplayer games. In Virtual Reality Continuum and Its Applications in Industry, ACM, 351-354.

Bredel, M., AND Fidler, M. 2010. A measurement study regarding quality of service and its impact on multiplayer online games. In SIGCOMM Workshop on Network and Systems Support for Games, NetGames '10, 1:1-1:6.

Claypool, M., and Claypool, K. 2006. Latency and player actions in online games. Communications of the ACM 49, 11, $40-45$.

Dick, M., Wellnitz, O., And Wolf, L. 2005. Analysis of factors affecting players' performance and perception in multiplayer games. In SIGCOMM Workshop on Network and System Support for Games, NetGames '05, 1-7.

Jehaes, T., De Vleeschauwer, D., Coppens, T., VAn Doorselaer, B., Deckers, E., NAudts, W., SPRUYT, K., AND SMETS, R. 2003. Access network delay in networked games. In SIGCOMM Workshop on Network and System Support for Games, NetGames '03, 63-71.

Jörg, S., Normoyle, A., And SAFOnova, A. 2012. How responsiveness affects players' perception in digital games. In Symposium on Applied Perception, SAP '12, 33-38.

MCCANn, J., AND POLlaRd, N. 2007. Responsive characters from motion fragments. Transactions on Graphics (SIGGRAPH) $26,3,6$.

Pavlovych, A., And Gutwin, C. 2012. Assessing target acquisition and tracking performance for complex moving targets in the presence of latency and jitter. In Graphics Interface, GI '12, $109-116$.

QuAX, P., Monsieurs, P., LAmotTe, W., De Vleeschaumer, D., And Degrande, N. 2004. Objective and subjective evaluation of the influence of small amounts of delay and jitter on a recent first person shooter game. In SIGCOMM Workshop on Network and System Support for Games, NetGames '04, 152-156.

UGC, 2014. TF2 Highlander league rules. United Gaming Clans website. http://www.ugcleague.com/rules_ tf2h.cfm. retrieved May 2014. 


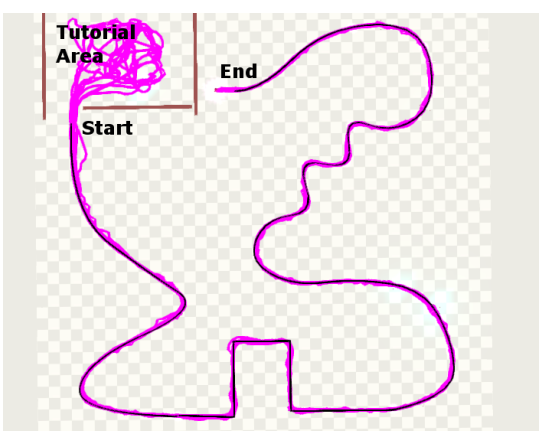

(a) Level 1, Condition $L_{0}$

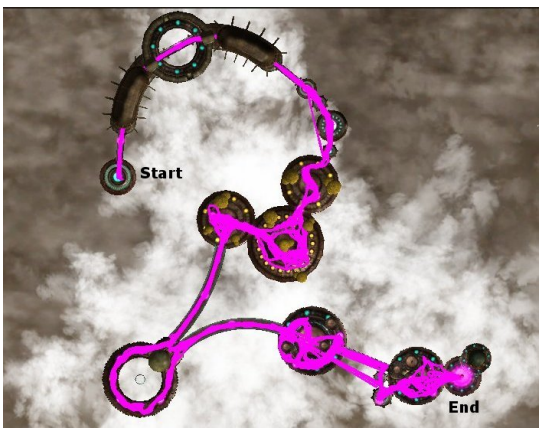

(d) Level 2, Condition $L_{0}$

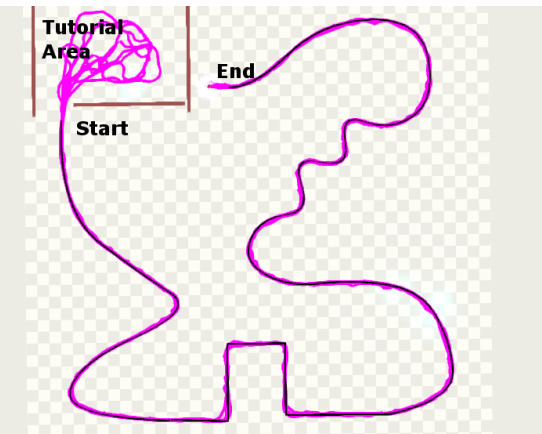

(g) Level 3, Condition $L_{0}$

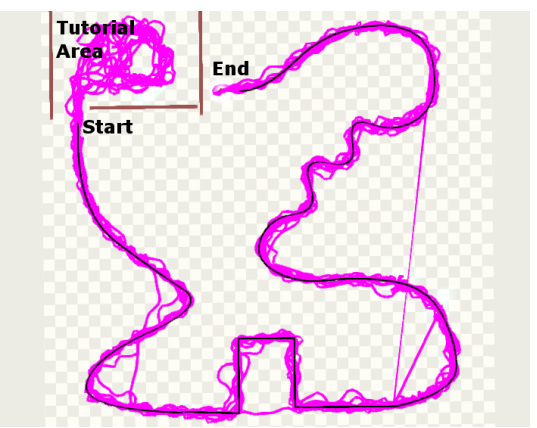

(b) Level 1, Condition $L_{500}$

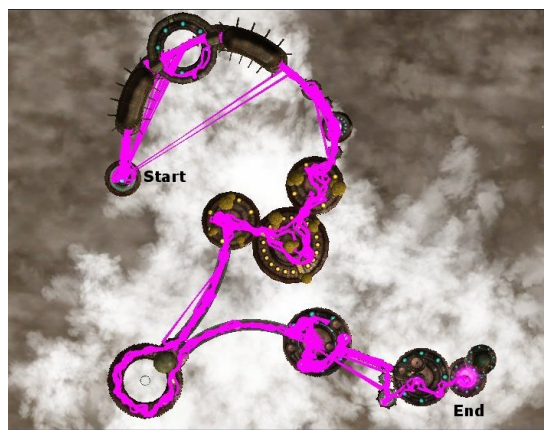

(e) Level 2, Condition $L_{500}$

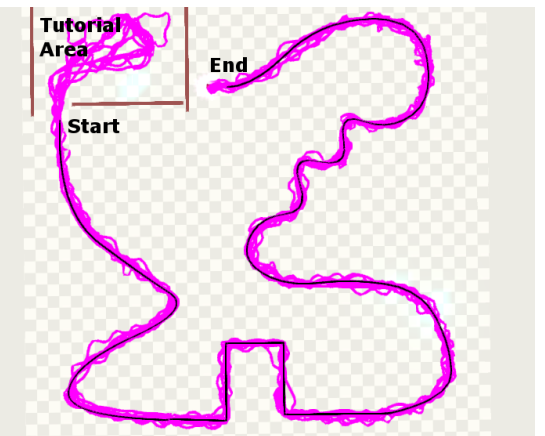

(h) Level 3, Condition $L_{500}$

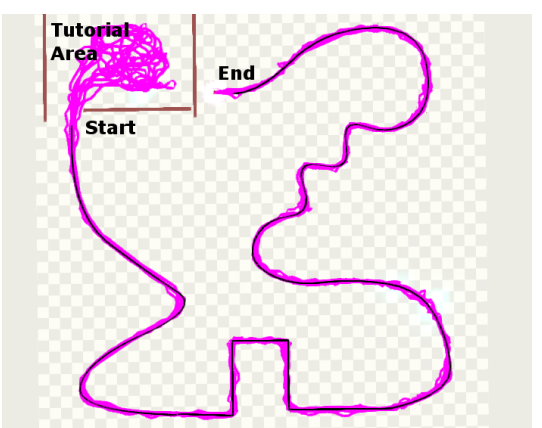

(c) Level 1, Condition $J_{150}$

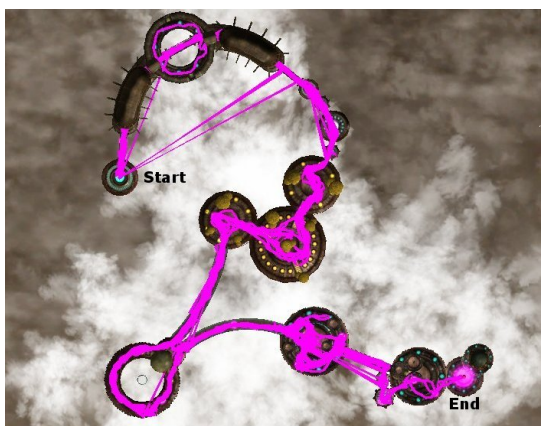

(f) Level 2, Condition $J_{150}$

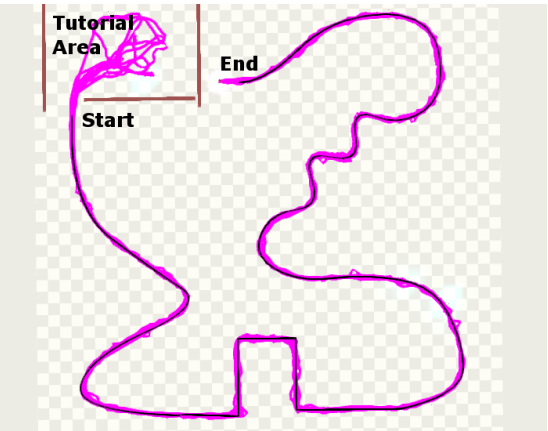

(i) Level 3, Condition $J_{150}$

Figure 3: Participant trajectories. This table compares the trajectories of participants for each level in three different conditions: $L_{0}$, $L_{500}$, and $J_{150}$. Straight lines indicate deaths where the player was teleported to a spawn point. In condition $L_{0}$ (leftmost column), where there is no latency or jitter, controlling the character is easiest and in these conditions, we see the smoothest trajectories. Note also that for level 2, all participants were able to navigate across the bridge at the top of the map as opposed to the other conditions where participants often opted to walk around. In condition $L_{500}$ (middle column), where there was a 500ms latency and no jitter, participants often "oversteered" the character due to the delay. Note that participants died more often, could not follow the path well, and for level 2, often did not finish the level (demonstrated by the lack of trajectories in the bottom right). In condition $J_{150}$, where we had a $200 \mathrm{~ms}$ latency and $150 \mathrm{~ms}$ jitter, participants again had a difficult time controlling the character, demonstrated by the increased number of deaths and lack of smooth trajectories. Comparing the first row and last row, we can see the learning effect. Participants spend less time in the tutorial area and follow the path more closely in level 3 shown in the last row. (Note: the maps for level 1 and level 3 have been desaturated to show the paths better) 